\title{
CD146: the unveiling of a pro-angiogenic netrin receptor
}

\author{
Cell Research (2015) 25:533-534. doi:10.1038/cr.2015.42; published online 7 April 2015
}

Netrin-1, a classic neuronal guidance cue, can promote angiogenesis under certain developmental and pathological conditions, but key receptors on vascular endothelium have remained elusive. A recent study published in Cell Research by Tu et al. reveals that CD146, an endothelial receptor of the immunoglobulin superfamily, binds netrin-1 with high affinity and may play an important role in regulating angiogenesis.

The anatomical similarity of the nervous and vascular systems has long been recognized, and it is now known that many of the neuronal guidance cues, such as netrins, slits, semaphorins and ephrins, also regulate angiogenesis the sprouting of new blood vessels. During neurogenesis axonal growth cones play a key navigational role, sensing local cues in the environment and relaying signals that culminate in axon extension or retraction. Similarly, endothelial tip cells direct new blood vessel sprouts during angiogenesis. Netrins, derived from the Sanskrit word 'netr' meaning 'one who guides', were first shown to promote the outgrowth of commissural axons in 1994 and are comprised of a family that includes three secreted members (netrins 1, 3, and 4) and two GPI-linked members (netrins G1 and G2). Netrin-1, the prototypical netrin, attracts or repulses growth cones depending on receptor status. Beginning in 2004, several published studies have revealed that netrin-1 also has proangiogenic or anti-angiogenic activity, the balance depending on ligand concentration and presumably the receptors expressed on the endothelium lining the vessels, although the identity of the pro- angiogenic netrin receptor has remained elusive [1-4].

To stimulate neuronal outgrowth, netrin-1 binds Deleted in Colorectal Cancer (DCC), a type I cell surface receptor of the immunoglobulin superfamily critical for neurogenesis (Figure 1). A related DCC family member, neogenin, also promotes axon guidance, whereas members of the uncoordinated 5 family (UNC5A-D) predominantly inhibit neurogenesis. Recent structural studies demonstrate that netrin- 1 is able to crosslink its receptors, with $\mathrm{DCC} /$ DCC homodimers promoting chemoattraction and DCC/UNC5 heterodimers promoting chemorepulsion [5]. In the vascular system UNC5B is highly expressed in endothelial cells during embryonic development and pathological angiogenesis and, like the role of UNC5s in repression of neurogenesis, may mediate netrin-1 antiangiogenic activity [3]. However, it has been unclear how the pro-angiogenic activity of netrin-1 is mediated because DCC and neogenin are not detected in most endothelium. The previous lack of a clear angiogenesis-promoting netrin receptor on proliferating vascular endothelium led Mehlen's group to propose that UNC5B could act as a dual purpose "dependence receptor" either stimulating or blocking endothelial apoptosis depending on ligand availability [6].

In an elegant study recently published in Cell Research, Tao Tu and coworkers from Xiyun Yan's laboratory provide compelling evidence that CD146, an endothelial cell surface receptor of the immunoglobulin superfamily (Figure 1) can stimulate angiogenesis in response to netrin-1 [7]. CD146 was first described in 1987 based on its elevated expression in malignant versus normal melanocytes, and is also called Melanoma Cell Adhesion Molecule (MCAM or Mel-CAM). Soon after its discovery, CD146 was also found to be widely expressed on vascular endothelium and smooth muscle. CD146 is elevated in some tumor types, including melanoma, prostate and breast cancers, and recent studies demonstrate that it can promote epithelial to mesenchymal transition. In cultured endothelial cells CD146 has been shown to promote migration, proliferation and tube formation [7]. Although CD146-null mice are viable, impaired tumor angiogenesis and tumor growth in these mice suggests that this receptor may play a critical role in pathological angiogenesis [8].

Tu et al. [7] show that netrin-1 directly binds CD146 with high affinity $(\mathrm{Kd}=1.3 \mathrm{nM})$, somewhat higher than its affinity for the repulsive netrin receptor UNC5B $(\mathrm{Kd}=5.1 \mathrm{nM})$. Through deletion analysis they show that the $\mathrm{V}$ domain of netrin-1, which contains three laminin-like EGF domains, plays an important role in binding. By analogy with data from the crystal structure of netrin-1 in complex with DCC [5], regions in both the $\mathrm{V}$ domain and the N-terminal VI domain of netrin-1 may bind CD146, potentially explaining the residual binding the authors observe with their $\mathrm{V}$ domain mutant. Netrin-1 treatment of endothelial cells resulted in a biphasic response with low doses $(50-200 \mathrm{ng} / \mathrm{mL})$ inducing proliferation, migration and tube formation and high doses (1 000-2 $000 \mathrm{ng} / \mathrm{mL})$ inhibiting these effects. Knockdown of CD146 inhibited the pro-angiogenic pheno- 


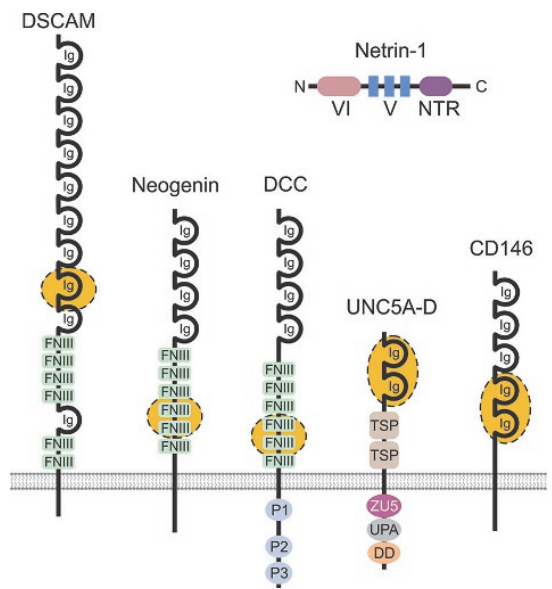

Figure 1 Receptors of the immunoglobulin superfamily that bind Netrin-1. Schematic representation of netrin-1 and its receptors. Netrin-1 contains an N-terminal laminin type $\mathrm{VI}$ domain, a $\mathrm{V}$ region containing three laminin-like EGF domains, followed by a C-terminal netrin-like domain (NTR). The $\mathrm{VI}$ and $\mathrm{V}$ domains play an important role in receptor binding. The receptor binding sites for netrin-1 have been mapped to the regions shown in yellow. DSCAM, Down's syndrome cell adhesion molecule; DCC, Deleted in Colorectal Cancer; DD, death domain; FNIII, fibronectin type III domain; Ig, immunoglobulin domain; TSP, thrombospondin type-1 domain; P1-3, conserved regions of the DCC cytoplasmic domain; UPA, UNC5, PIDD and ankyrin domain; ZU5, zona occludens 5 domain.

types observed at low doses, whereas knockdown of UNC5B prevented the anti-angiogenic effects at high doses. Importantly, the authors demonstrate the importance of CD146 for angiogenesis in vivo using a CD146 neutralizing antibody and conditional knockout mice that lack CD146 in endothelium. Furthermore, the authors demonstrate that knockdown of either CD146 or netrin-1 leads to a similar defect in parachodal vessel formation during zebrafish development.

Tu et al. find that addition of netrin-1 to endothelial cells is able to induce the formation of an unusually stable CD146 dimer that remains intact following exposure to the anionic detergent SDS. The authors also find that VEGFR2, the main VEGF receptor involved in VEGF-mediated angiogenesis, is rap- idly phosphorylated upon addition of netrin-1 to serum-starved endothelial cells, which in turn activates signaling through ERK and p38 kinase, while CD146 knockdown prevents these effects. Netrin-1 was unable to bind VEGFR2 directly, and further studies are needed to determine exactly how these pathways intersect and the biological significance of these interactions in vivo. Like any landmark study, the work raises many new questions. For example, is the pro-angiogenic role of netrin-1 through CD146 completely dependent on the increased VEGFR2 signaling, or can netrin-1 also stimulate angiogenesis independent of VEGFR2? Is VEGF required for the rapid VEGFR2 phosphorylation in response to netrin-1 - for example, can netrin-1 mobilize matrix-bound VEGF or promote intracrine signaling, or does the purified recombinant netrin-1 contain trace amounts of VEGF? Can CD146 form heterodimers with other netrin receptors, like UNC5B? Can netrin signal through CD146 in non-endothelial cells, such as pericytes and smooth muscle cells that are also CD146 positive?

Another important area concerns the role of netrin/CD146 signaling in pathological angiogenesis. While CD146 has been found to be widely expressed in adult normal vascular endothelium through gene expression profiling and using various anti-CD146 antibodies, for example the s-Endo-1 or the $\mathrm{P} 1 \mathrm{H} 12$ antibodies, the AA98 mouse anti-human CD146 antibody developed in Yan's laboratory appears to preferentially label CD146 on tumor-associated endothelium compared to normal endothelium $[9,10]$. AA98, which binds the same region of CD146 as netrin-1, prevents dimerization of the receptor $[7$, 11]. If particular regions of CD146 are uniquely exposed during pathological angiogenesis, for example surfaces on the newly produced monomeric receptor that are normally masked upon ligand binding and dimerization, then this could potentially be exploited for specific targeting of tumor angiogenesis.
Although the knockdown studies in zebrafish and cultured endothelial cells provide important evidence for the role of this receptor in angiogenesis, so far no clear developmental angiogenesis defect in CD146 and netrin-1 knockout mice has been reported. One possible explanation is ligand or receptor redundancy. In that regard, netrin-3 and netrin- 4 , which have not yet been tested for CD146 binding, have also been found to have angiogenic activity in vivo. Integrins, which are widely expressed on endothelium, have also been implicated as netrin-binding receptors [12]. Another possibility is that the netrin/CD146 interactions are more important for angiogenesis associated with wound repair, ischemia and other pathological conditions than for development. If so, then targeting the netrin/CD146 axis could have important therapeutic implications for various angiogenesis-dependent diseases.

\section{Brad St Croix ${ }^{1}$}

${ }^{1}$ Tumor Angiogenesis Section, Mouse Cancer Genetics Program, National Cancer Institute (NCI), NIH, Frederick, MD 21702, USA

Correspondence: Brad St Croix E-mail: stcroix@ncifcrf.gov

\section{References}

1 Wilson BD, Ii M, Park KW, et al. Science 2006; 313:640-644.

2 Lu X, Le Noble F, Yuan L, et al. Nature 2004; 432:179-186.

3 Larrivee B, Freitas C, Trombe $\mathrm{M}$, et al. Genes Dev 2007; 21:2433-2447.

4 Park KW, Crouse D, Lee M, et al. Proc Natl Acad Sci USA 2004; 10:16210-16215.

5 Finci LI, Kruger N, Sun X, et al. Neuron 2014; 83:839-849.

6 Castets M, Coissieux MM, Delloye-Bourgeois C, et al. Dev Cell 2009; 16:614-620.

7 Tu T, Zhang C, Yan H, et al. Cell Res 2015; 25:275-287.

8 Zeng Q, Wu Z, Duan H, et al. Protein Cell 2014; 5:445-456.

9 Yan X, Lin Y, Yang D, et al. Blood 2003; 102:184-191.

10 Seaman S, Stevens J, Yang MY, et al. Cancer Cell 2007; 11:539-554.

11 Zheng C, Qiu Y, Zeng Q, et al. Int J Biochem Cell Biol 2009; 41:2163-2172.

12 Nikolopoulos SN, Giancotti FG. Cell Cycle 2005; 4:e131-e135. 